\title{
The Text-Message Discourse Completion Task: A Method for the Collection of Text-Messages
}

\author{
Andrew Albritton \\ Missouri State University \\ Management Department \\ College of Business \\ 901 S. National \\ Springfield, MO 65897, USA
}

\begin{abstract}
The process of collecting text-messages for scholarly investigation poses researchers with a number of challenges related to communicative context, privacy, and data transferal. This paper explains a data collection method that largely overcomes these challenges. The method is a form of Discourse Completion Task in which study participants are asked to send from their mobile phones the text-messages they would send in certain hypothetical scenarios. While similar methods have been used in some previous studies, this paper presents the first full explanation and justification of the text-message DCT method, and includes an explanation of the how the method was used successfully in a study of punctuation marks in text-messages.
\end{abstract}

Keywords: Discourse Completion Task, mobile communication, text-message

\section{Introduction}

This paper contains a description of a data collection method that can, in a relatively easy and cost-effective manner, provide socially contextualized and digitally composed text-messages to researchers studying communicative and linguistic practices in text-based mobile communication. The method is, in short, a form of Discourse Completion Task (DCT) in which study participants are given hypothetical social situations and are asked to send the text-messages they would send in those situations.

Similar methodology has been used in some previous studies. The DCT was previously used in a study of textmessaging by Plester, Wood, and Joshi (2009), who, although they did not use the term DCT in their article, did employ the basic DCT technique in conjunction with a questionnaire for the purpose of examining correlations between children's literacy levels and their uses of non-standard spelling in text-messages. Plester, Wood, and Joshi's (2009) study appears to be the first use of a DCT to study digital communication. It must be mentioned that although it is not stated explicitly, it is evident that these researchers had the students handwrite their text-message responses. Behnam, Hamed, and Asli (2012), in a study of digital condolences in English and Persian, used DCTs via email and asked respondents to send, in email responses, messages that they would send via text-message in certain given scenarios. Marzuki and Walter (2013) examined apologies in English and Malay text-messages. In their study, they sent the DCT prompts and received the replies via text-messages.

While the DCT method was used in these previous studies, the method is not fully discussed or justified in the papers that presented the studies. The novelty of this paper thus lies not in the presentation of the text-message DCT method, but in the full explanation and justification of the method. This discussion includes a detailed description of how the method was used in a study of punctuation mark function in text-messages. It is hoped that, in reading this paper, researchers will see the benefits and relatively easy operationalization of the text-message DCT and embrace it when appropriate for their studies of language use in text-messages.

\section{Challenges of Gathering Text-Message Data}

The collection of text-message data for scholarly investigation poses researchers with a number of unique challenges. In the first place, there is a difficulty related to text-message context. In order to acquire a robust understanding of the meanings within text-messages, a researcher requires not only the text-messages themselves, but also information concerning the social situations in which those text-messages were sent. As will be discussed in the next section, current methods do not allow for the easy collection of contextual data.

In the second place, there is a challenge related to the confidentiality of people's text-messages. The text-message medium is often used for interpersonal and emotive purposes (Harkin, 2003; Kasesniemi \& Rautiainen, 2002; Lenhart, Ling, Campbell, \& Purcell, 2010; Ling \&Yttri, 2002; Oksman \& Turtiainen, 2004). 
Sometimes, it is used as a way to share private or sensitive information; Lenhart et al. (2010) found that "Threequarters of texting teens use text messaging to exchange information privately - with more than a quarter doing this daily or several times a day. Another three-quarters of text-using teens also say they have long message exchanges by text to discuss important personal matters" (online, p. 5, "Why teens text", para. 3).

Text-messages are not absolutely private, since they can potentially be seen by people other than the intended recipient(s). Kelly et al. (2012) reported that the college students who participated in their focus groups recognized the fact that records of text-message exchanges could potentially be shown to other people; the students viewed this as a way that their privacy could be invaded. However, notwithstanding the semi-privacy of the medium, text-messages are still used as a way to communicate intimate messages that are intended only for a single person (or a limited group of people). For this reason, people may be hesitant to share their messages with a researcher, even if they understand that the collected data will not be connected to them in the researcher's final report (Lyddy et al., 2014).

In the third place, there is a challenge related to the way that text-messages can be submitted to the researcher. When a person is willing to give his or her text-messages to a researcher, it is important that those messages be transmitted and saved in such a way that they can be seen as they were composed on the mobile phone. Having research respondents transcribe past text-messages by hand onto paper is clearly not the best method for gathering data: In projects where such a method is used, a number of which are cited below, there could be transcription errors as a result of oversight; or the respondent might intentionally change certain elements of the transcribed text-messages in order to make them less personally impeaching, or more orthographically and grammatically standard; or, indeed, the respondent may be inclined to oblige the researcher with florid uses of non-standard orthography. Furthermore, transcriptions of messages containing emojis would be problematic, in that respondents would be required to sketch the emojis. Moreover, in methods in which hypothetical text-messages are composed and sent, inscribing messages by hand is still not the best option. De Jonge and Kemp (2012), in a study of the "textisms" (or text-message abbreviations) of Australian young people, had students transpose sentences into "textese" (the language of text-messaging, as characterised by certain features of orthographic inventiveness [see Shortis, 2007]). The scholars had one group of students type their textese messages into their phones and then handwrite exactly what they had typed, and had another group simply handwrite the textese messages without typing them into their phones. The authors found that the use of textisms was similar in both groups, indicating that young people can indeed write in valid textese by hand - or at least can write textisms as they normally would type them. However, even though De Jonge and Kemp's (2012) typing-then-handwriting method is an interesting idea, and even though their conclusions point towards an ability on the part of young people to handwrite the textisms of textese, there is no doubt that the collection of text-messages actually typed into mobile phones and sent from mobile phones is preferable to the collection of text-messages written by hand. People cannot be expected to write with a pencil or pen exactly what they would type with a keyboard. And, furthermore, in research settings in which respondents are asked to handwrite what they would normally type, the respondents may be discouraged from using emojis (since they would have to draw them), when in normal circumstances they would readily insert such pictographs into their text-messages. In order to obtain text-message data of the best quality, the text-messages collected should be written on the respondents' mobile phones and should be sent digitally, such that they can be read as originally composed.

\section{Methods Used in Previous Text-Message Studies}

Previous text-message studies have used a number of methodologies. Here, these methodologies will be briefly discussed, and it will be shown how the method explained in this paper fills in a gap in the methodological field.

Focus groups have been used as a means of better understanding text-messaging in several investigations, including those of Grinter and Eldridge (2001), Kasesniemi and Rautiainen (2002), Ling and Yttri (2002), Harkin (2003), Oksman and Turtiainen (2004), Lenhart et al. (2010), and Kelly et al. (2012). Similarly, individual interviews have been used as a way to examine text-messaging practices: Kasesniemi and Rautiainen (2002) and Oksman and Turtiainen (2004) used data collected via this method. Questionnaires and surveys have also been utilized in the investigation of text-messaging; data collected via these methods was used by Grinter and Eldridge (2001), Kasesniemi and Rautiainen (2002), Faulkner and Culwin (2005), Lenhart et al. (2010), Harrison and Gilmore (2012), and Morrill, Jones, and Vaterlaus (2013). The methodologies of focus groups, interviews, and questionnaires/surveys can be very helpful in understanding what people think about their text-messaging practices and the text-messaging practices of others. These methods do not, however, generate actual text-messages for analysis.

Diary and logging studies have also been used by text-messaging researchers. When this type of methodology is employed, respondents transcribe some or all of the text-messages they transmit and/or receive during a given period of time, while also notating information concerning those text-messages, such as the times when they were sent or received. 
Data collected by means of this method was used by Grinter and Eldridge (2001) (who did not actually elicit transcriptions of the text-messages), Kasesniemi and Rautiainen (2002), Faulkner and Culwin (2005), and Baron and Ling (2011). The diary or logging method is one in which the context of messages can, to some degree, be established. However, in most cases, it would not be practicable for respondents to give particularly detailed descriptions of the social contexts of the text-messages they wrote down. Further, now that many people are sending copious textmessages on a daily basis, asking a person to keep track of all of his or her text-messages - or even a majority of them - in a diary or log is probably not reasonable. Another shortcoming of the diary/log method is that people have the ability to alter or omit their messages. As was mentioned above, many text-messages contain personal and sensitive material, some of which (or, indeed, much of which) the participant in the study may not desire for the researcher to see. Further, because in diary studies the messages are being written down (or potentially typed into a computer), there could be transcription errors. So, while this method provides more context for individual text-messages, it still has weaknesses related to transposition inaccuracies and the modification and exclusion of messages.

Other text-messaging studies have used text-message corpora. In some cases - such as in the studies of Thurlow and Brown (2003), Baron and Ling (2011), and Lyddyet al. (2014) - relatively small corpora have been constructed by asking respondents to transcribe text-messages; it need not be further elaborated that such transcription is not ideal. Some scholars have built much larger corpora. Tagg (2009) made a corpus of 11,067 text-messages. She collected the text-messages from friends, family, and an online message board. The text-messages that were taken from friends and family were written by hand or were typed into a template in an Excel spreadsheet, while those collected online were typed onto the message board. How (2004) constructed a corpus of 10,117 text-messages using volunteers, the Internet, and email solicitation. Work on How's (2004) corpus was recommenced in 2010 (Chen \&Kan, 2013), and people from around the world could contribute their English or Mandarin text-messages to this corpus in different ways: Users of Android mobile phones could use an app to upload their messages; users of Nokia mobile phones could create .csv files of their sent text-messages and then email those files; and users of other types of phones could go to a webpage and type their text-messages into fields. It was requested for ethical reasons that people who volunteered their textmessages for this corpus send only text-messages they had personally written and transmitted. Fairon and Paumier (2006) sent out public requests that people send copies of their text-messages to a toll-free number; using this method, they built a corpus of 30,000 French text-messages. Treurniet, De Clercq, van den Heuvel, and Oostdijk (2012) made a corpus of Dutch text-messages. Their collection methods were similar to those of Chen and Kan (2013) in that they allowed people to submit messages in the following ways: Android users could send their text-messages with an app; Nokia and iPhone users could upload sent-message back-up files to an online mailbox or dropbox; and people could also go online and type their messages into fields on a webpage.

In the construction of the corpora cited in the previous paragraph, a number of collection methodologies were employed. It is clear that the superior methods were those that did not require any transcription, but rather allowed people to transmit their text-messages directly from their phones to the people who were building the corpora. For, even when transcription is done on a keyboard, there is the possibility of error or intentional alteration on the part of the submitter; and even if the messages are copied and pasted, there is still the opportunity for intentional modification of the message. No matter the collection method, however, all of these corpora demonstrate a shared weakness - the lack of context. This is not to say that corpora such as these cannot be put to many salutary scholastic purposes. They allow for important linguistic patterns to be analyzed; and furthermore, it should be observed that some contextual elements naturally become evident because of the semantic content of text-messages. However, context is of vital importance in evaluating many aspects of language use, and the more that can be known about the communicative context of a message, the better.

All of the methods discussed in the preceding paragraphs can be used to gather excellent and helpful data, and yet they all evince certain shortcomings. Self-reporting methods, while they can provide good data concerning how people think and feel about text-messages, do not provide any actual text-messages to study and interpret; and there is always the possibility in such studies that people may tell the researcher one thing about their behavior while doing something else. Diary and logging methods, while they can provide transcripts of text-messages and some contextual information about those text-messages, lend themselves to transcription infelicities, and can put an unrealistic burden on prolific senders of text-messages; and, importantly, the contextual information contained within diaries or $\operatorname{logs}$ can be impoverished or altered. Text-message corpora, while they can help to illumine linguistic patterns of note, do not provide contextual information beyond what is inferable from the semantic content of the text-messages and any metadata that may be collected. 


\section{The DCT Method}

For the studies in which communicative practices in text-messages will be investigated, actual text-messages are desirable, as well as at least some information concerning the social contexts of those messages. In order to obtain such data, the DCT data collection technique may be employed, and the messages generated by respondents can be composed on phones and sent to phones or email addresses. In general, the DCT method is one in which respondents are given hypothetical circumstances and asked what they would say (or write in a text-message, in our case) in those circumstances. For studies of text-messaging, respondents would be asked to write the text-message on their phones and send them to researchers' phones or email addresses.

Traditionally, the DCT elicitation method has been operationalized in the following manner: Investigators present participants with a certain social circumstance and ask the participants to record what they would say in that circumstance. The method thus allows for the researcher to exert control over both the type of utterance that is elicited and the context of that utterance. A researcher using the DCT is able not only to obtain, for instance, general examples of expressions of gratitude (i.e., the speech act of thanking), but is able to obtain examples of expressions of gratitude in highly controlled hypothetical communicative contexts, thereby enabling the attainment of more refined understandings of how gratitude is articulated. For this reason, DCTs "have been widely and successfully used in the study of speech acts... and speech events (e.g. asking the time)" (O'Keeffe, Clancy, \&Adolphs, 2011, p. 21). The DCT method was first developed by Blum-Kulka (1982) in a study that investigated the speech act production of language learners as compared to native speakers. Since then, DCTs have often been used in studies concerning language learning and interlanguage pragmatics. Beebe and Waring (2004), for instance, used a DCT to investigate the differences in the ways that lower-level learners and more proficient learners of English used adverbials to create tone. Tanck (2002) used a DCT to compare the ways in which English speakers and English learners produce refusals and complaints. Other scholars who have implemented DCTs to investigate interlanguage pragmatics include Blum-Kulka and Olshtain (1984), Chen (1996), and Eisenstein and Bodman (1986). One vital theme that runs through the DCT literature is the notion that learners of a new language must be instructed not merely in the vocabulary and grammar of the language, but also in the pragmatics of the language, such that their utterances are both grammatically and communicatively competent. DCTs can, amongst many other potential functions, operate to show where students of certain languages are lacking in their understandings of the pragmatics of a new language, and these weaknesses can then be addressed by scholars and educators.

Some scholars have investigated the DCT method itself. Billmyer and Varghese (2000) found that DCT respondents who are given more detailed prompts reply with more robust responses. Sasaki (1998) compared role-playing exercises with DCTs and found that role-plays led to responses that were lengthier and more diverse in formulation than those obtained through DCTs. Beebe and Cummings (1995) evaluated how language gathered through DCTs is different than naturally occurring language. They found that the language data collected by means of DCTs is usually lacking in the richness and variety of naturally occurring language, but they also concluded that DCTs are an excellent way to discover general patterns in language usage. Schauer and Adolphs (2006) looked at DCT data as compared with corpus data, and found that, while corpora can provide abundant and sundry examples of language usage, DCTs can provide examples from very specific circumstances; they make the point that corpora, although they are frequently gargantuan in size, may not always have the specific examples of language formulas a researcher is searching for. In those cases when examples of a very specific type of linguistic behavior are being sought, DCTs are an auspicious methodological option. Furthermore, Schauer and Adolphs (2006), who were concerned with how data from DCTs and corpora could be used in the language-learning context, pointed out that DCT data can demonstrate newer word meanings and patterns that may not have emerged so clearly in corpus data - especially if the corpus being used is dated, even in the slightest degree. But Schauer and Adolphs (2006) did warn that DCTs are sorely lacking when it comes to issues of conversation analysis. The DCT does not provide actual language from an exchange between people, and data from DCTs is therefore necessarily one-sided and lacking in the conversational nuances that are produced in interpersonal interactions. Tanck (2002), who, as mentioned above, put the DCT to good use in a study of the linguistic patterns of refusals and complaints among native English speakers and English learners, offered a similar critique of the DCT (referring to it by its other name, the "Discourse Completion Test"): "The Discourse Completion Test, while a timeefficient instrument, may not be the best way to obtain authentic data. Subjects are writing, not speaking, and have the opportunity to contemplate and change their responses, something that is less possible in a naturalistic spoken setting" (p. 14).

It should be noted that Tanck (2002) here brings up a methodological deficiency intrinsically entailed in having people respond in a medium different than the one they would normally respond in - and different than the one being studied. Traditionally, DCTs have required respondents to write what they would say. 
This is a primary weakness of the method, but one, it seems, that could be at least to some extent overcome if respondents were asked to actually speak aloud their response; these responses could be recorded - perhaps both aurally and visually - and the method would thereby increase in validity. It is also worth highlighting Tanck's (2002) assertion that in normal conversation there is less time to think about responses than when participating in a DCT. Moreover, when engaging in in-person discourse, a person is faced with another human being in all of his or her complexity; in engaging in a DCT exercise, a person is faced with a piece of paper, and so DCTs, in their normal paper-based presentation, cannot fully provide the paralinguistic and nonverbal cues that are such an integral part of human relations.

Given this literature, a number of general conclusions can be drawn concerning how best to implement DCTs. First, it should be realized that the DCT is a method by which certain general patterns can be investigated; the full variety and irregularity of language in use is avowedly not seen in DCT data. In addition, data obtained through the DCT method will not (in most cases) have a great deal of information concerning how language operates to mediate conversation. With these limitations in mind, however, the DCT can certainly be put to efficacious use: It can be used as a way to look at the common patterns of language as they occur in specific contexts.

The text-message DCT not only allows for an understanding of the context of the text-messages that are collected, but it also allows for the researcher to fabricate those contexts him or herself. Furthermore, the concern over the privacy of respondents is bypassed using this method. Some of the weaknesses of the DCT method will admittedly persist, however, when implementing a text-message DCT. Primarily, the text-message DCT will suffer from a lack of full discussions, which means that the data collected will not exhibit the richness of forms and the bounty of conversational information that would normally be seen in naturally occurring text-messages.

Some of the weaknesses of the traditional DCT method will not apply to the text-message DCT. As Tanck (2002) pointed out in her criticism of the method, the rapid-fire, intuitive nature of in-person conversation is lacking in a traditional DCT; but these characteristics of in-person conversation are often absent in text-messaging, even in those conversations in which messages are flying back and forth with relative quickness. In the realm of text-messaging, people are not usually in the presence of their digital interlocutor, so they always have some time to formulate a response, and they are not on the spot to try and come up with responses immediately. Any concerns regarding intermedia discrepancies are likewise allayed, since the method allows for the study of actual text-messages that were composed and sent from a mobile phone.

The text-message DCT method is therefore one that can be used to obtain socially contextualized text-message data that exhibit general communication patterns.

\section{Implementation of the Text-Message DCT}

The text-message DCT method proposed in this paper was implemented successfully in a study of punctuation mark function in text-messages; it was conducted as a component of a $\mathrm{PhD}$ research project concerned with punctuation mark function in digital discourse in general. The study's respondents were students in an introductory computer class at a Midwestern American university. They were given a research instrument (Appendix) that, on one side of a single sheet of paper, included an informed consent form and four DCTs, as well as one request for demographic information. Students used their own phones to send their text-message responses and participated at their own expense.

The study in which the DCTs were used gave special attention to how punctuation marks can convey emotional and relational meanings in text-messages. The first DCT's purpose was to elicit a congratulatory response; it read, "You just heard that a good friend got a job he or she had been wanting. What would you text your friend? Please text your message to [email address 1]." The second DCT's purpose was to elicit a consolatory response; it read, "You just heard that your friend did not get a job he or she had applied for. What would you text your friend? Please textyour message to [email address 2].' The third DCT's purpose was to elicit a message for a more dubious emotional circumstance; it read, "You just heard that your friend got a new job, but you're not sure what he or she thinks about it. What would you text your friend? Please text your message to [email address 3]." The fourth DCT's purpose was to elicit a less emotional and more task-based message for the purpose of contrast; it read, "You are part of a small group working on a project for a class. You think the group should have a meeting. What would you text the group's leader? Please text your message to [email address 4]." The instrument requestedthat respondents send a fifth text-message with demographic information; it read, "Please text your age and gender to [email address 5]." The messages were copied out of the email inboxes to which they were sent and were pasted into two Word documents; the first organized the responses according to the DCT, and the second according to the respondent who sent the message.

In total, 136 text-messages were collected for the study. (The study was qualitative and interpretive, investigating punctuation marks from a rhetorical and semiotic perspective, and thus a larger amount of data was not necessary for the initial study.) 
The data obtained via the text-message DCT illuminated interesting patterns in punctuation use, as well as some other linguistic patterns of note. The data provided answers to a number of research questions, and other researchers investigating text-message practices could undoubtedly implement this method of data collection with similar results.

There were some irregularities in the data that should be noted. Some respondents in the study wrote text-message responses which seem to indicate that they were responding to a text-message from the friend in question, as opposed to initiating a conversation; this fact does not present any problems to the study - whether or not the message is primary or secondary in the given exchange, it can still be used to analyze how meaning is conveyed in the message. However, in future studies it may, in certain circumstances, be best to make clear whether the elicited text-message is a first or second message in an exchange. If it is a second message, the first message should be given in the DCT. One respondent, who participated in only one of the DCTs, sent his or her response to the email address set up for DCT 1, but it was clearly intended for DCT 2 (it read, "Awhh:-/ you can find a better job anyway!"). Two respondents had typos in their messages and sent follow-up messages with corrections marked with an asterisk (a symbol used in digital communiqués to indicate a rectification of a typo in a previous message); these follow-up messages, incidentally, are themselves an interesting part of the data. One respondent sent a text-message that was delivered in two emails, presumably because he or she was sending the message via the Short Message Service (SMS) and the number of characters in the message exceeded the 160-character limit. Another respondent's message was cut-off mid-sentence; perhaps this respondent pressed send before the message was complete. These irregularities are enumerated simply to illustrate some of the minor issues that can arise when using the text-message DCT. As long as the researchers using the method are conscientious when they organize the collected messages for analysis, there should not be any insuperable problems with the data.

One further issue that may come up in future text-message DCTs is that of how to view emojis in the collected data. The Unicode Consortium has introduced standardized coding for numerous emojis. However, the visual renderings of the respective emoji vary depending on the operating system of the device being used to view them. Researchers should be aware of these discrepancies. In order to know the exact appearance of emoji used by respondents, researchers would need to ask respondents to indicate the device they used when writing their messages; then, using data in a chart from Unicode about how the disparate operating systems render emoji (available at http://unicode.org/emoji/charts/fullemoji-list.html), researchers could see the precise pictograms the respondents used.

This operationalization of the method is, of course, not the only one that researchers could use. For example, if practicable, researchers could send text-message prompts and receive responses back via text-message, as in the study of Marzuki and Walter (2013).

\section{Further Applications of the Method}

DCTs could be used in a number of interesting ways to further explore text-messaging. They could be deployed in projects similar to the one described above - projects that are concerned with punctuation in text-messaging, affective displays in text-messaging, context-based differences in the formality of language in text-messaging, etc. Furthermore, it will be recalled that DCTs were first used to study speech acts, and they could easily be put to this purpose in the digital realm. For instance, it might be asked, how are certain speech acts in certain situations realized in textmessages? This and other similar queries could be answered using text-message DCTs. DCTs could also be used in exploring interlanguage pragmatics in the digital sphere.

Moreover, DCTs need not be limited to the study of text-messages. DCTs could be used to collect emails and study how people construct these digital missives in different contexts; interesting studies could be accomplished using this method in which stylistic and structural aspects of emails are examined. DCTs could even be used to study IM chatting, although, admittedly, role-plays, in which the researcher or other participants are active interlocutors, may be a better choice for these studies, given the normally more synchronous nature of IM conversation. And it is certainly worthy of note that, regardless of the purpose of the study or the genre of digital discourse under consideration, the digital DCT will always have the advantage of not invading the privacy of respondents (as long, of course, as the DCT does elicit replies that necessitate the inclusion of personal information).

The technique of having respondents send text-messages to email accounts could also be used in studies that did not implement DCTs. Experimental studies might use a similar method. Or, researchers could construct a minor corpus in which young people select some messages they have previously sent in certain social circumstances, and copy and paste those messages into new transmissions which are then sent to email addresses; in cases such as these, even though respondents would be asked to copy and paste their messages, there would still be the possibility that respondents would not copy and paste their submissions or would alter the submissions in some way; all the same, there is no question that the email technique is better than the paper-based transcription technique. 
Furthermore, now that smartphones are so popular, it would be possible for research participants to take screenshots of past texting conversations and email those to researchers. If this screenshot collection technique were implemented, informed consent would need to be acquired from all of the people involved in the texting exchange, since the screenshot would necessarily display both sent and received messages.

\section{Conclusion}

DCTs are a propitious method of data collection in the study of text-messages, and, potentially, in the study of other forms of digital discourse: They allow for the collection of digitally written and transmitted messages with an understanding of the social context in which messages are composed and sent, and yet they do not call for respondents to disclose personal or sensitive information. DCTs are elicitation instruments, and thus the data gathered via their implementation cannot be considered natural language; however, DCTs do allow for a careful control of the communicative context under consideration, and studies have shown that DCTs can bring forth general and conventional patterns of language use. The text-message DCT was successfully implemented, with relative ease and effectiveness, in a study of punctuation practices in text-messages, and could, in the future, be adapted to many different kinds of studies of digital communication.

\section{References}

Baron, N., \& Ling, R. (2011). Necessary smileys \& useless periods. Visible Language, 45(1/2), 45-67.

Beebe, L.M., \& Cummings, M.C. (1995). Natural speech act data versus written questionnaire data: How data collection method affects speech act performance. In S.M. Gass\& J. Neu (Eds.), Speech acts across cultures: Challenges to communication in a second language (pp. 65-86). New York: Mouton de Gruyter.

Beebe, L.M., \& Waring, H. (2004). The linguistic encoding of pragmatic tone: Adverbials as words that work. In D. Boxer \& A. Cohen (Eds.), Studying speaking to inform second language learning (pp. 228-249). Clevedon, England: Multilingual Matters.

Behnam, B., Hamed, L. A. A., \&Asli, F. G. (2013). An investigation of giving condolences in English and Persian via short messages. Procedia-Social and Behavioral Sciences, 70, 1679-1685.

Billmyer, K., \& Varghese, M. (2000). Investigating instrument-based pragmatic variability: Effects of enhancing discourse completion tests. Applied Linguistics, 21(4), 517-552.

Blum-Kulka, S. (1982). Learning to say what you mean in a second language: A study of the speech act performance of learners of Hebrew as a second language. Applied Linguistics, 3(1), 29-59.

Blum-Kulka, S., \&Olshtain, E. (1984). Requests and apologies: A cross-cultural study of speech act realization patterns (CCSARP). Applied Linguistics, 5(3), 196-213.

Chen, H. (1996). Cross-cultural comparison of English and Chinese metapragmatics in refusal. (Doctoral dissertation). Retrieved from http://files.eric.ed.gov/fulltext/ED408860.pdf

Chen, T. \&Kan, M. Y. (2013). Creating a live, public short message service corpus: The NUS SMS Corpus. Language Resources and Evaluation, 47(2), 299-355.

De Jonge, S., \& Kemp, N. (2012). Text-message abbreviations and language skills in high school and university students. Journal of Research in Reading, 35(1), 49-68.

Eisenstein, M., \&Bodman, J.W. (1986). 'I very appreciate': Expressions of gratitude by native and non-native speakers of American English. Applied Linguistics, 7(2), 167-185.

Fairon, C., \&Paumier, S. (2006). A translated corpus of 30,000 French SMS. Proceedings of the Fifth International Conference on Language Resources and Evaluation (LREC 2006), 351-354.

Faulkner, X., \&Culwin, F. (2005). When fingers do the talking: A study of text messaging.Interacting with Computers, 17(2), 167-185.

Grinter, R. E., \& Eldridge, M. (2001). y dotngrs luv 2 txt msg? In W. Prinz, M. Jarke, Y. Rogers, K. Schmidt \& V. Wulf (Eds.), Proceedings of the seventh European conference on computer-supported cooperative work ECSCW '01, Bonn, Germany (pp. 219-238). Dordrecht, The Netherlands: Kluwer Academic Publishers.

Harkin, J. (2003). Mobilisation: The growing public interest in mobile technology. London: Demos.

Harrison, M. A., \& Gilmore, A. L. (2012). U txt WHEN? College students' social contexts of text messaging. Social Science Journal, 49(4), 513-518.

How, Y. (2004). Analysis of SMS efficiency. (Undergraduate thesis). National University of Singapore. Retrieved from http://wing.comp.nus.edu.sg:8080/SMSCorpus/data/pubs/howYijueThesis.pdf

Kasesniemi, E., \&Rautiainen, P. (2002). Mobile culture of children and teenagers in Finland. In J. E. Katz \& M.A. Aakhus (Eds.), Perpetual contact: Mobile communication, private talk, public performance (pp. 170-192). Cambridge: Cambridge University Press. 
Kelly, L., Keaten, J. A., Becker, B., Cole, J., Littleford, L., \&Rothe, B. (2012). It's the American lifestyle!: An investigation of text messaging by college students. Qualitative Research Reports in Communication, 13(1), 19.

Lenhart, A., Ling, R., Campbell, S., \& Purcell, K. (2010). Teens and mobile phones. Retrieved from http://www.pewinternet.org/Reports/2010/Teens-and-Mobile-Phones.aspx

Ling, R. \&Yttri, B. (2002). Hyper-coordination via mobile phones in Norway. In J. E. Katz \& M.A. Aakhus (Eds.), Perpetual contact: Mobile communication, private talk, public performance (pp. 139-169). Cambridge: Cambridge University Press.

Lyddy, F., Farina, F. Hanney, J., Farrell, L., \& Kelly O’Neill, N. (2014). An analysis of language in university students' text messages. Journal of Computer-Mediated Communication, 19(3), 546-561.

Marzuki, E., \& Walter, C. (2013). English and Malay text messages and what they say about texts and cultures. Open Journal of Modern Linguistics, 3(04), 295-304.

Morrill, T. B., Jones, R. M., \&Vaterlaus, J. (2013). Motivations for text messaging: Gender \& age differences among young adults. North American Journal of Psychology, 15(1), 1-16.

O’Keeffe, A., Clancy, B., \&Adolphs, S. (2011). Introducing pragmatics in use. London: Routledge.

Oksman, V., \&Turtiainen, J. (2004). Mobile communication as a social stage: Meanings of mobile communication in everyday life among teenagers in Finland. New Media \& Society, 6(3), 319-339.

Plester, B., Wood, C., \& Joshi, P. (2009). Exploring the relationship between children's knowledge of text message abbreviations and school literacy outcomes. British Journal of Developmental Psychology, 27(1), 145-161.

Sasaki, M. (1998). Investigating EFL students' production of speech acts: A comparison of production questionnaires and role plays. Journal of Pragmatics, 30(4), 457-484.

Schauer, G., \&Adolphs, S. (2006). Expressions of gratitude in corpus and DCT data: Vocabulary, formulaic sequences, and pedagogy. System, 34(1), 119-134.

Shortis, T. (2007). Gr8 txtpectations: The creativity of text spelling. English Drama MediaJournal,8, 21-26.

Tagg, C. (2009). A corpus linguistics study of SMS text messaging. (Doctoral dissertation). The University of Birmingham, Birmingham, UK. Retrieved from http://etheses.bham.ac.uk/253/1/Tagg09PhD.pdf

Tanck, S. (2002). Speech act sets of refusal and complaint: A comparison of native and non-native English speakers' production. American University, Washington, DC. Retrieved from http://observer.american.edu/cas/tesol/pdf/upload/WP-2004-Tanck-Speech-Act.pdf

Thurlow, C., \& Brown, A. (2003). Generation txt? Exposing the sociolinguistics of young people's text-messaging. Discourse Analysis Online, 1(1).

Treurniet, M., De Clercq, O., van den Heuvel, H., \&Oostdijk, N. (2012). Collection of a corpus of Dutch SMS. 8th International Conference on Language Resources and Evaluation Conference (LREC-2012), 2268-2273.

\section{Appendix}

\section{Text Message Study}

Conducted by [author]

[author's email address]

Thank you for taking the time to consider participating in my study. This study is being conducted as part of my research for a $\mathrm{PhD}$ degree from the University of Nottingham. My research is about the language of text messages. I am also investigating new ways to collect text message data. Your participation in this study is entirely voluntary. Participation should take about ten minutes. You do not have to complete all of the tasks, and you may stop participating at any time. Your answers and phone numbers will be kept strictly confidential. If you are under the age of 18, please do not participate.

This study will ask you to send a total of five text messages to five respective email addresses. (Text messages can be sent to email addresses just as they are sent to another phone - just type the email address in place of a phone number.) Standard text messaging rates do apply. If your phone plan requires you to pay for individual text messages and you do not wish to do so, please do not participate.

If you are willing to participate, please sign this form, then continue on to the instructions below.

Participant Signature

\section{Date}


Below you will be given made-up situations and will be asked to send the text message you would normally send in that given situation. Please write the text messages just as you would in the given situation. If you would normally use the recipient's name in the message, feel free to use a name of your choice.

1. You just heard that a good friend got a job he or she had been wanting. What would you text your friend? Please text your message to textresearch1@gmail.com.

2. You just heard that your friend did not get a job he or she had applied for. What would you text your friend? Please text your message to textresearch2@gmail.com.

3. You just heard that your friend got a new job, but you're not sure what he or she thinks about it. What would you text your friend? Please text your message to textresearch3@ gmail.com.

4. You are part of a small group working on a project for a class. You think the group should have a meeting. What would you text the group's leader? Please text your message to textresearch4@gmail.com.

5. Please text your age and gender to textresearch5@gmail.com.

Thank you very much for your participation. If you have questions about this study or are interested in the study's findings, please feel free to contact me at [author's email address]. 